\title{
INFLUENCE OF COSINE CORRECTOR AND UAS PLATFORM DYNAMICS ON AIRBORNE SPECTRAL IRRADIANCE MEASUREMENTS
}

\author{
Juliane Bendig ${ }^{1}$, Deepak Gautam ${ }^{1}$, Zbyněk Malenovský1,2, Arko Lucieer ${ }^{1}$ \\ ${ }^{1}$ School of Technology, Environments and Design, University of Tasmania, Hobart, TAS 7005, Australia \\ ${ }^{2}$ School of Biological Sciences, University of Wollongong, Wollongong, NSW 2522, Australia
}

\begin{abstract}
Measuring solar-induced chlorophyll fluorescence from smallsized Unmanned Aircraft Systems (UAS) can potentially fill the scaling gap between ground-based and airborne/spaceborne observations. These measurements require well calibrated, high-spectral resolution spectroradiometers and precise measurements of vegetation radiance and incoming solar irradiance. Here we present a system equipped with a spectroradiometer with a split optical path that measures incoming irradiance through a cosine corrector/diffuser. The objectives of this study are to characterise cosine corrected solar irradiance measurements with regard to sensor homogeneity and possible offset from an ideal cosine response. We further suggest a methodology to calculate a corrected zenith angle that accounts for changing sensor orientation due to pitch, roll and heading of the UAS platform during flight. We found that the cosine corrector is sufficiently homogeneous, thus measurements are independent of UAS heading. The response follows the cosine law for zenith angles, however, the sensor significantly underestimated irradiance for zenith angles $>10^{\circ}$, with overall cosine errors ranging from 0.991 to 1.229 . Yet, typical in-flight platform pitch and roll angles produce a zenith angle offset of up to $6^{\circ}$ in low wind conditions. Cosine sensor measurements corrected for the zenith angle offset and the cosine error resulted in a $1.7 \%$ change in irradiance.
\end{abstract}

Index Terms - spectroradiometer, solar-induced chlorophyll fluorescence, unmanned aircraft system, sun zenith angle, irradiance cosine corrector

\section{INTRODUCTION}

Spectroradiometers with a very high spectral resolution (2$3 \mathrm{~nm}$ ) enable estimates of steady state solar-induced chlorophyll fluorescence (SIF), which can act as nondestructive estimates of plants' photosynthetic effiency. Measurements with imaging and non-imaging sensors are acquired on the leaf to canopy scale with sensors mounted on various platforms. Up to now, mostly non-imaging spectroradiometers have been deployed on UAS, enabling flexible, spatially detailed observations that can provide valuable insight in upscaling SIF observations to larger scales, i.e. satellite resolutions. How- ever, these observations are technically challenging due to the need for: i) accurately locating the spectroradiometer footprint on the ground and ii) measuring ambient changes in sunlight radiation during a flight. Different solutions have been suggested for the latter challenge: i) averaging illumination through readings of spectralon panels before and after flight [1], ii) logging irradiance on a second, ground-based spectroradiometer with an attached cosine corrector/diffuser [2], or iii) spliting the optical pathway with a channel switching mechanism [3]. The last type, which is also presented here, has the advantage of frequent downwelling irradiance measurements. However, these measurements may be influenced by the UAS in-flight movements. The cosine corrector was, due to technical limitations, hard-mounted to the UAS airframe at the highest possible position. The downward looking channel was mounted on a high precision gimbal, which has been calibrated for boresight and lever-arm offset to maximise geometric accuracy of the downward looking spectroradiometer footprint. Adding a second, equaly precise gimbal for mounting the upward looking cosine sensor would add an extra computation complexity and might exceed the payload capacity of the airframe. Attaching both spectroradiometer channels to the same gimbal, e.g. with an extension stick, was unfeasible due to the length of the optical cables and the design of the multi-rotor airframe with a risk of damage by rotor blades. Therefore, we rather opted for characterization and correction of the cosine corrector's changing orientation during a UAS flight in relation to the sun position. Cosine correctors are designed to follow Lambert's cosine law, which states that the radiant intensity on a given surface is proportional to the cosine of the solar zenith angle of incidence [4]. The real angular response of a cosine corrector often differs from the cosine law, having a tendency to underestimate solar irradiance, which is known as the cosine error [5]. This underestimation is more significant under high zenith angles in the morning and afternoon hours. Since intensity of SIF emitted by plants follows a diurnal course, characterization of cosine corrector measurements at high zenith angles is crucial. Therefore, we investigated the angular response of our system with the specific objectives of: i) testing azimuthal independence of cosine corrector readings (i.e. verifying the diffuser homogeneity), ii) determining the 


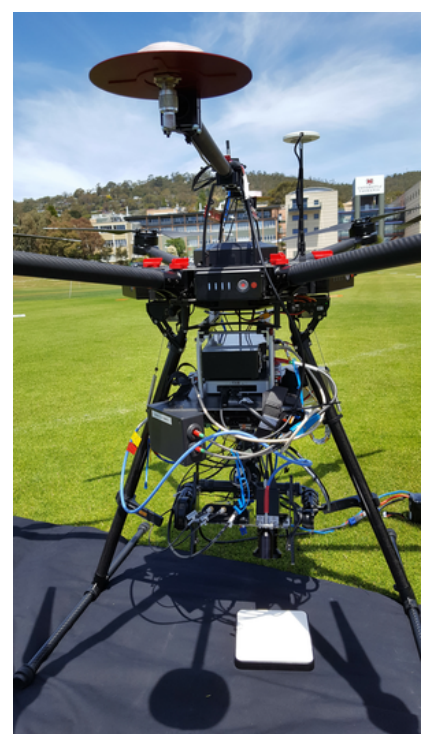

(a)

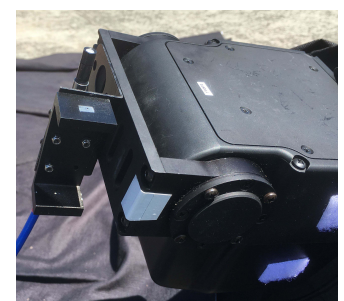

(b)

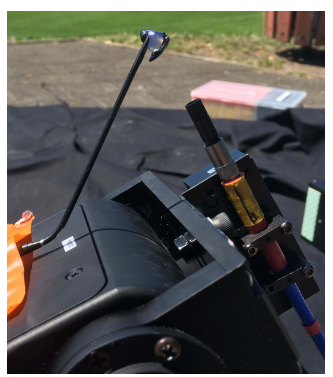

(c)
Fig. 1. (a) UAS airframe and payload, (b) robotic head with optical fiber and cosine corrector, parallel mounted pinhole sun-finder, (c) shading disk.

zenith-dependent offset from a perfect cosine response, and iii) correcting the relative zenith angle between the cosine sensor and sun.

\section{MATERIALS AND METHODS}

The UAS-based spectroradiometer system presented here was designed to measure SIF with a special focus on a high accuracy characterization and geo-positioning of signal footprint.

\subsection{Spectroradiometer}

A QE Pro spectroradiometer (Ocean Optics, Inc., USA) with a spectral range of $500-877 \mathrm{~nm}$, a spectral sampling interval of $0.33 \mathrm{~nm}$, full width at half maximum (FWHM) of $0.8 \mathrm{~nm}$, and 18-bit digital range (signal to noise ratio of a single acquisition 1000:1, and dynamic range 85000:1) was integrated onto a multi-rotor UAS airframe. The system has a two channel input for measuring upwelling reflected radiance (VEG channel) and downwelling solar irradiance (IRR channel) and optical shutters to switch between the channels. The VEG channel has a field of view (FOV) of about $8^{\circ}$, restricted by a Gershun tube. The IRR channel has a hemispherical $\left(180^{\circ}\right)$ cosine corrector attached (CC-3, Ocean Optics). The VEG channel is mounted on a gimbal (Foxtech Eagle Eye DSLR aerial gimbal, China) and the IRR channel is hard-mounted to an antenna boom on top of the airframe. The system has been radiometrically and spectrally calibrated to output radiance values.

\subsection{UAS sensor payload}

To track the orientation of the VEG channel during a flight, an inertial measurement unit (IMU: Advanced Navigation Spatial Dual, Sydney, Australia) was mounted on the gimbal next to the VEG channel. To measure the position, we mounted a custom built antennae boom with a dual frequency dual antennae (Antcom G5Ant-1.9A4-XTB-1, California, USA) on top of the UAS airframe (DJI Matrice 600 (Dà-Jiāng Innovations Science and Technology Co., Ltd., China), Fig. 1). An IMU/Global Navigation Satellite System (GNSS) (Lord MicroStrain 3DM-GX3-35, Vermont, USA) was firmly attached to the antennae boom alongside the IRR channel. Geometrical offset between IMU and VEG channel and IMU and antennae were measured and corrected. A GNSS base station was used during flight to provide a correction signal. The spectroradiometer and on-board positioning systems were triggered and synchronised by an on-board data logger.

\subsection{Experiment 1: Homogeneity or azimuth angle de- pendence}

We tested the homogeneity of the cosine corrector under different zenith and azimuth angles, i.e. a multi-angular direct irradiance, in a controlled experiment. Direct irradiance was produced by an artificial, stable light source LS-150 Xenon Arc Lamp Source (ABET Technologies Inc., Connecticut, USA) with an Ushio UXL-150-MO lamp (USHIO Inc., Japan). A black tube attached to the output port restricted the size of the light beam. The IRR channel was fixed on a robotic head (FLIR pan and tilt unit PTU-D48: FLIR Systems Inc., Oregon, USA) and aligned with the center of the light beam at a fixed distance. Changing zenith angles were simulated by turning the robotic head with respect to the light source from $-25^{\circ}$ to $25^{\circ}$ in $0.5^{\circ}$ steps. Two readings were averaged per angle. The experiment was repeated three times with the cosine corrector rotated by $90^{\circ}$ each time to simulate different azimuth angles.

\subsection{Experiment 2: Zenith angle dependence}

In this experiment we reused the setup from experiment 1 (see 2.3), which was adapted for natural sunlight as proposed in [6]. A cosine corrector measurement per wavelength is defined as:

$$
E=I \cos (z) f(z)+D \bar{f}
$$

where $E$ is the global irradiance collected by the sensor, $I$ is the direct component, depending on cosine of zenith $z$, and $D$ is the diffuse component. $f(z)$ and $\bar{f}$ are the instrument specific factors. $\bar{f}$ depends on the angular distribution of diffuse irradiance, which is assumed to be isotropic. If $f(z)$ is known, $\bar{f}$ can be calculated as suggested in $[5,7]$. We further assume that global irradiance and the ratio between the direct and diffuse components do not change significantly during the 
whole experiment. The cosine receptor's relative cosine error may be calculated from:

$$
\text { cosine error }=\frac{I_{z}}{I_{0} \cos (z)}
$$

where $I_{0}$ is the direct irradiance at zenith angle 0 and $I_{z}$ at a particular zenith angle $z$. A perfect instrument would have a cosine error equal to 1 . The IRR channel was aligned with the sun by using a pinhole camera principle, similar to the FluoWat leaf clip [8] (Fig. 1). Five spectral readings were recorded at angles from $-50^{\circ}$ to $50^{\circ}$ with a step of $5^{\circ}$. At the end of each cycle, the robotic head was returned to the starting position to check for the sun's movement during the measurement. A second set of diffuse irradiance measurements was carried out instantly, with the IRR channel shadowed by a shading disk of the cosine corrector's size $(6 \mathrm{~mm})$. The difference between global and diffuse equals the direct component. Global irradiance measurements took $3 \mathrm{~min}$. Diffuse irradiance measurements took $10 \mathrm{~min}$, as the position of the shading disk had to be manually adjusted. Two sets of measurements were acquired under sunny conditions at the University of Tasmania (17-18 December 2017) between 14:40 and 15:15 local time.

\subsection{Experiment 3: Flight dynamics - zenith correction}

The influence of UAS platform roll, pitch and heading angles on the irradiance measurement was investigated during a test flight undertaken at the University of Tasmania on 23 December 2016 at 12:50 local time. The 15 min long cross-grid flight was carried out in stable, sunny conditions with light winds. For the duration of the flight, we calculate the angular difference between the cosine corrected IRR channel and the sun position, referred to as corrected zenith angle. First, we retrieved the sun position defined by the zenith and azimuth angle for that particular day, time, and location. The zenith and azimuth angles of the sun position were used to define a unit direction vector, which points from the cosine corrector to the sun as presented in Equation 3:

$$
\overrightarrow{\operatorname{sun}}=[\cos \alpha \sin \beta, \sin \alpha \sin \beta,-\cos \beta]^{\mathrm{T}}
$$

where $\overrightarrow{\operatorname{sun}}$ is a unit direction vector that points to the direction of the sun from the cosine corrector. $\alpha \in\left[0^{\circ}, 360^{\circ}\right]$ and $\beta \in\left[0^{\circ}, 90^{\circ}\right]$ are the azimuth and zenith angles, respectively, which define the sun position. Consequently, the pointing angle of the IRR channel as measured by the MicroStrain IMU was used to parametrize a unit direction vector $\overrightarrow{i r r}$, which represents the pointing direction of the IRR channel as follows:

$$
\overrightarrow{i r r}=R_{m s} \times \overrightarrow{z e n}
$$

where, $R_{m s}$ is the attitude matrix parametrized using the orientation of the MicroStrain IMU and $\overrightarrow{z e n}$ is a unit direction vector pointing vertically up. The dot product was applied to the above two vectors, one pointing to the sun $(\overrightarrow{s u n})$ and another pointing along the cosine corrected IRR channel $(\overrightarrow{i r r})$, to yield the corrected zenith angle $(\angle z e n)$ as presented in Equation 5:

$$
\angle z e n=\arccos \left|\frac{\overrightarrow{s u n} \cdot \overrightarrow{i r r}}{\|\overrightarrow{s u n}\| \cdot\|\overrightarrow{i r r}\|}\right| .
$$

\section{RESULTS \& DISCUSSION}

Raw spectral signatures were converted from digital numbers to radiance. Figure 2 shows the radiance at four receptor rotations, i.e. azimuth angles averaged for selected wavelengths. The wavelengths were selected based on relevance for chlorophyll fluorescence retrieval $(687,756,760 \mathrm{~nm})$ and photochemical reflectance index (PRI) $(531,570 \mathrm{~nm})$. The highest radiance was recorded at $-20^{\circ}$ and decreased with increasing zenith angles, which shows different radiant intensities within the light beam. Rotating the cosine corrector produced the same pattern, indicating homogeneous response of the cosine corrector, which is independent from azimuth. Data collected within experiment 2 was averaged for negative and positive angles (e.g. $-50^{\circ}$ and $50^{\circ}$ ), i.e. left and right of zenith $0^{\circ}$, for both observational days (coefficient of variation: 0.01). Figure 3 shows the relative cosine response of the corrector for direct irradiance averaged for the selected wavelengths (see above). Across all 1040 bands of the spectroradiometer, the relative cosine response varied by $0.18 \%$ with a maximum of $1.47 \%$ at $50^{\circ}$. The incoming irradiance is underestimated by the instrument with the error increasing with zenith angle, similar to the results in $[4,6]$. The relative cosine error (Equation 2) for the direct component ranged from 1.002 at $5^{\circ}$ to 1.229 at $50^{\circ}$. We used the methodology suggested in $[4,5]$ to derive the cosine error for the global component (0.991 1.185) and applied the correction factor to the data described in 2.5 in order to compensate the cosine corrector's underperformance. The influence of the UAS platform movements was expressed in the corrected zenith angle (see 2.5) and resulted in a mean zenith offset of $1.9^{\circ}$ with a maximum of $5.7^{\circ}$. Table 1 shows the resulting changes in irradiance before and after applying the cosine correction factor. The irradiance underestimation was up to $3.2 \%$, with an average around $1.7 \%$.

Table 1. Radiance $\left(\mathrm{Wm}^{-2} \mathrm{~nm}^{-1} \mathrm{sr}^{-1}\right)$ recorded at selected wavelengths during the test flight described in 2.5 before and after cosine response correction (mean of $\mathrm{n}=32$ ).

\begin{tabular}{c|c|c|c|c|c} 
wl & $\mathbf{5 3 1}$ & $\mathbf{5 7 0}$ & $\mathbf{6 8 7}$ & $\mathbf{7 5 6}$ & $\mathbf{7 6 0}$ \\
\hline no corr. & 0.608 & 0.569 & 0.337 & 0.085 & 0.419 \\
cos corr. & 0.616 & 0.578 & 0.344 & 0.087 & 0.427 \\
\% change & 1.281 & 1.531 & 2.050 & 1.821 & 1.821
\end{tabular}




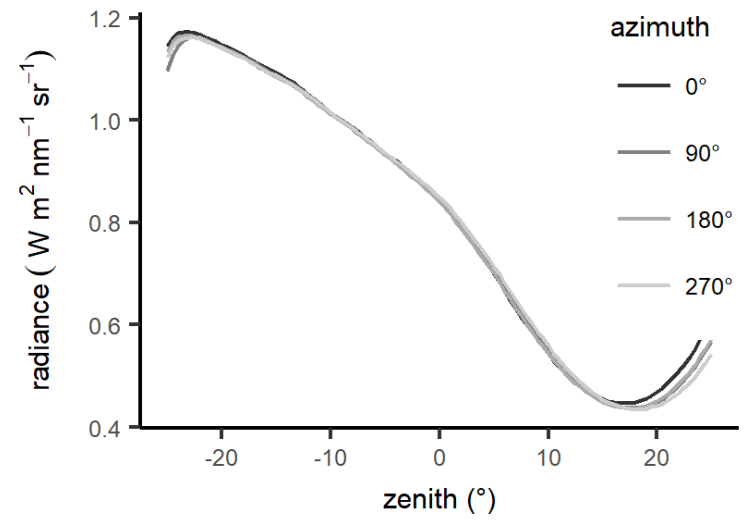

Fig. 2. Cosine corrector response (mean of five selected wavelengths) of an artificial, stable light source for four azimuth angles, $x$-axis: zenith angle, $y$-axis: radiance

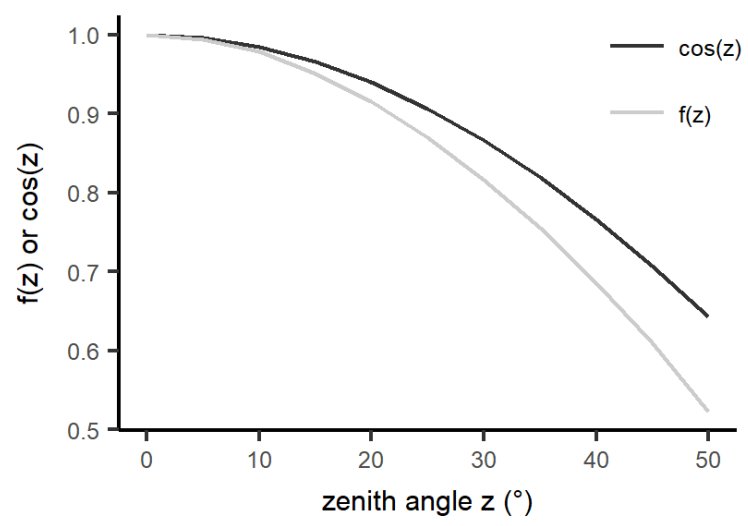

Fig. 3. Instrument specific cosine response and perfect cosine response depending on zenith angle (mean of five selected wavelengths); $\mathrm{x}$-axis: zenith angle $z$, y-axis: $f(z)$ and $\cos (z) * f(z)$

\section{CONCLUSION}

A UAS-mounted spectroradiometer with a split pathway enables frequent measurements of upwelling radiance and downwelling irradiance. The cosine corrector, which we used for irradiance measurements, provided a homogeneous, i.e. azimuth independent, spectral response. Its angular response followed the cosine law, but underestimated irradiance for increasing zenith angles. A correction function was, therefore, developed to account for this under-performance. Furthermore, we suggested a relative zenith angle correction to account for platform movements during the UAS flight. Relative zenith angles deviated up to $6^{\circ}$ from solar zenith angles for a typical flight in low winds. Correction for cosine receptor error and relative zenith angle resulted in differences of up to $3.2 \%$ and on average $1.7 \%$ in measured irradiance.
Hence, these corrections are important for increasing the accuracy of solar-induced chlorophyll fluorescence retrievals, especially when attempting diurnal measurements, where high zenith angles occur.

\section{REFERENCES}

[1] Andreas Burkart, Sergio Cogliati, Anke Schickling, and Uwe Rascher, "A novel uav-based ultra-light weight spectrometer for field spectroscopy," IEEE Sensors Journal, vol. 14, no. 1, pp. 62-67, Jan. 2014.

[2] Roberto Garzonio, Biagio Di Mauro, Roberto Colombo, and Sergio Cogliati, "Surface reflectance and suninduced fluorescence spectroscopy measurements using a small hyperspectral uas," Remote Sensing, vol. 9, no. 5, pp. 472, 2017.

[3] Alasdair Mac Arthur, Iain Robinson, Micol Rossini, Neville Davis, and Ken MacDonald, "A dual-field-ofview spectrometer system for reflectance and fluorescence measurements (piccolo doppio) and correction of etaloning," in Proceedings of the Fifth International Workshop on Remote Sensing of Vegetation Fluorescence, 2014, pp. 22-24.

[4] Jose Manuel Andujar Marquez, Miguel Ángel Martínez Bohórquez, Jonathan Medina Garcia, and Francisco Jose Aguilar Nieto, "A new automatic system for angular measurement and calibration in radiometric instruments," Sensors, vol. 10, no. 4, pp. 3703-3717, 2010.

[5] Alkiviadis F. Bais, Stelios Kazadzis, Dimitrios Balis, Christos S. Zerefos, and Mario Blumthaler, "Correcting global solar ultraviolet spectra recorded by a brewer spectroradiometer for its angular response error," Appl. Opt., vol. 37, no. 27, pp. 6339-6344, Sep 1998.

[6] Christopher Kuchinke and Manuel Nunez, "Spectral dependence in the cosine response of broadband uv instruments," Journal of Geophysical Research: Atmospheres, vol. 106, no. D13, pp. 14287-14300, July 2001.

[7] Manuel Antón, Antonio Serrano, María L. Cancillo, José M. Vilaplana, Victoria E. Cachorro, and Julian Gröbner, "Correction of angular response error in brewer uv irradiance measurements," Journal of Atmospheric and Oceanic Technology, vol. 25, no. 11, pp. 2018-2027, Nov. 2008.

[8] Luis Alonso, Luis Gómez-Chova, Joan Vila-Francés, Julia Amorós-López, Luis Guanter, Javier Calpe, and Josh Moreno, "Sensitivity analysis of the fraunhofer line discrimination method for the measurement of chlorophyll fluorescence using a field spectroradiometer," in Geoscience and Remote Sensing Symposium, 2007. IGARSS 2007. IEEE International. IEEE, 2007, pp. 3756-3759. 